\title{
Improved semi-local convergence of the Gauss-Newton method for systems of equations
}

\author{
Ioannis K. Argyros ${ }^{\mathrm{a}}$ and Santhosh George ${ }^{\mathrm{b}^{*}}$ \\ ${ }^{a}$ Department of Mathematical Sciences, Cameron University, Lawton, OK 73505, USA \\ ${ }^{\mathrm{b}}$ Department of Mathematical and Computational Sciences, National Institute of Technology Karnataka, India-575 025 \\ *Corresponding author E-mail: sgeorge@nitk.ac.in
}

\section{Article Info}

Keywords: Gauss- Newton method, Newton's method, Semi-local convergence, Least squares problem 2010 AMS: 65H10, 65G99, 65K10, 47H17, 49M15

Received: 8 June 2018

Accepted: 13 September 2018

Available online: 30 September 2018

\begin{abstract}
Our new technique of restricted convergence domains is employed to provide a finer convergence analysis of the Gauss-Newton method in order to solve a certain class of systems of equations under a majorant condition. The advantages are obtained under the same computational cost as in earlier studies such as [5, 14]. Special cases and a numerical example are also given in this study.
\end{abstract}

\section{Introduction}

Let $\Omega \subseteq \mathbb{R}^{n}$ be open. Let $F: \Omega \rightarrow \mathbb{R}^{m}$ be continuously Fréchet- differentiable. The problem of approximating least squares solutions $x^{*}$ of the nonlinear problem

$$
\min _{x \in \Omega}\|F(x)\|^{2}
$$

is very important in computational mathematics. The least squares solutions of (1.1) are stationary points of $Q(x)=\|F(x)\|^{2}$. A lot of problems arising in applied sciences and in engineering can be expressed in a form like (1.1). For example in data fitting $n$ is the number of parameters and $m$ is the number of observations. Other examples can be found in [6, 16, 19] and the references therein. The famous Gauss-Newton method defined by

$$
x_{k+1}=x_{k}-F^{\prime}\left(x_{k}\right)^{\dagger} F\left(x_{k}\right), \text { for each } k=0,1, \cdots,
$$

where $x_{0}$ is an initial point and $F^{\prime}\left(x_{k}\right)^{\dagger}$ the Moore-Penrose inverse of the linear operator $F^{\prime}\left(x_{k}\right)$ has been used extensively to generate a sequence $\left\{x_{k}\right\}$ converging to $x^{*}[1]-[6],[8,10,20,14,15,17]$.

In the present paper, we are motivated by the work of Goncalves and Oliveira in [14] (see also [12], [13]) and our works in [1, 2, 3, 4, 6, 7, 8]. These authors presented a semi-local convergence analysis for the Gauss-Newton method (1.2) for systems of nonlinear equations where the function $F$ satisfies

$$
\left\|F^{\prime}(y)^{\dagger}\left(I_{\mathbb{R}^{m}}-F^{\prime}(x) F^{\prime}(x)^{\dagger}\right) F(x)\right\| \leq k\|x-y\| \text { for each } x \text { and } y \in \Omega,
$$

where $k \in[0,1)$ and $I_{\mathbb{R}^{m}}$ denotes the identity operator on $\mathbb{R}^{m}$. Their semilocal- convergence analysis is based on the construction of a majorant function (see condition $\left(h_{3}\right)$ ). Their results unify the classical results for functions involving Lipschitz derivative [6, 7, 16, 18] with results for analytical functions $(\alpha$-theory or $\gamma$-theory) $[9,11,15,17,19,20]$.

We introduce a center majorant function (see $\left(c_{3}\right)$ ) which is a special case of the majorant function that can provide more precise estimates on the distances $\left\|F^{\prime}(x)^{\dagger}\right\|$. Then, we find a domain where the iterates lie which is more precise than in the aformentioned studies. This leads to "smaller" majorant functions yielding to weaker sufficient convergence conditions; more precise error estimates on the distances $\left\|x_{k+1}-x_{k}\right\|,\left\|x_{k}-x^{*}\right\|$ and an at least as precise information on the location of the solution.

The rest of the paper is organized as follows: The semi-local convergence analysis of the Gauss-Newton method is presented in Section 2. Special cases and numerical examples are given in the concluding Section 3. 


\section{Semi-local convergence analysis}

In this section we present the semi-local convergence analysis of the Gauss-Newton method. Let $R>0$. Denote by $B\left(x_{0}, R\right), \bar{B}\left(x_{0}, R\right)$ the open and closed balls in $\mathbb{R}^{n}$, respectively with center $x_{0} \in \mathbb{R}^{n}$ and radius $R$. We shall use the hypotheses denoted by $(\mathscr{C})$.

$\left(c_{0}\right)$ Let $B\left(x_{0}, R\right) \subseteq \mathbb{R}^{n}$ and $F: B\left(x_{0}, R\right) \rightarrow \mathbb{R}^{m}$ be continuously Fréchet- differentiable.

$\left(c_{1}\right)$ continuously differentiable functions $f_{0}:[0, R) \longrightarrow \mathbb{R}, f:\left[0, R^{*}\right) \rightarrow \mathbb{R}$

$$
\left\|F^{\prime}\left(x_{0}\right)^{\dagger}\right\|\left\|F^{\prime}(x)-F^{\prime}\left(x_{0}\right)\right\| \leq f_{0}^{\prime}\left(\left\|x-x_{0}\right\|\right)-f_{0}^{\prime}(0) \text { for each } x \in B\left(x_{0}, R\right)
$$

and

$$
\left\|F^{\prime}\left(x_{0}\right)^{\dagger}\right\|\left\|F^{\prime}(y)-F^{\prime}(x)\right\| \leq f^{\prime}\left(\|y-x\|+\left\|x-x_{0}\right\|\right)-f^{\prime}\left(\left\|x-x_{0}\right\|\right) \text { for each } x, y \in B\left(x_{0}, R^{*}\right)
$$

with $\|y-x\|+\left\|x-x_{0}\right\|<R^{*}$ where $R_{0}:=\sup \left\{t \in[0, R]: f_{0}^{\prime}(t)<0\right\}$. Set

$$
R^{*}:=\min \left\{R_{0}, R\right\} .
$$

$\left(c_{2}\right)$

$$
\left\|F^{\prime}(y)^{\dagger}\left(\mathbb{I}_{\mathbb{R}^{m}}-F^{\prime}(x) F^{\prime}(x)^{\dagger}\right) F(x)\right\| \leq \kappa\|x-y\| \text { for each } x \text { and } y \in B\left(x_{0}, R^{*}\right),
$$

where $\kappa \in[0,1)$.

$\left(c_{3}\right)$ Set $\eta=\left\|F^{\prime}\left(x_{0}\right)^{\dagger} F\left(x_{0}\right)\right\|>0, F^{\prime}\left(x_{0}\right) \neq 0$.

$$
\operatorname{rank}\left(F^{\prime}(x)\right) \leq \operatorname{rank}\left(F^{\prime}\left(x_{0}\right)\right) \neq 0 \text { for each } x \in B\left(x_{0}, R^{*}\right) .
$$

$\left(c_{4}\right)$

$$
\begin{gathered}
f_{0}(0)=f(0)=0, f^{\prime}(0)=f_{0}^{\prime}(0)=-1 \\
f_{0}(t) \leq f(t) \text { and } f_{0}^{\prime}(t) \leq f^{\prime}(t) \text { for each } t \in\left[0, R^{*}\right) .
\end{gathered}
$$

(c $\left.c_{5}\right) f_{0}^{\prime}, f^{\prime}$ are convex and strictly increasing.

Let $\mu \geq 0$ be such that $\mu \geq-\kappa f^{\prime}(\eta)$ and define $\varphi_{\eta, \mu}:\left[0, R^{*}\right) \rightarrow \mathbb{R}$ by

$$
\varphi_{\eta, \mu}(t)=\eta+\mu t+f(t) .
$$

(c $\left.c_{6}\right) \varphi_{\eta, \mu}(t)=0$ for some $t \in\left[0, R^{*}\right)$.

$\left(c_{7}\right)$ For each $s, t, u \in\left[0, R^{*}\right)$ with $s \leq t \leq u$

$$
t+\frac{\varphi_{\eta, \mu}(u)}{f_{0}^{\prime}(u)} \leq u+\frac{\varphi_{\eta, \mu}(t)-\varphi_{\eta, \mu}(s)-\varphi_{\eta, \mu}^{\prime}(s)(t-s)}{f_{0}^{\prime}(t)}
$$

The majorizing iteration $\left\{r_{k}\right\}$ for $\left\{x_{k}\right\}$ is given by

$$
r_{0}=0, r_{k+1}=r_{k}-\frac{\varphi_{\eta, \mu}\left(r_{k}\right)}{f_{0}^{\prime}\left(r_{k}\right)}
$$

The corresponding iteration $\left\{t_{n}\right\}$ used in [14] is given by

$$
t_{0}=0, t_{k+1}=t_{k}-\frac{\bar{\varphi}_{\eta, \mu}\left(t_{k}\right)}{g^{\prime}\left(t_{k}\right)}
$$

where $\bar{\varphi}_{\eta, \mu}(t)=\eta+\mu t+g(t)$, continuously differentiable function $g:[0, R) \longrightarrow \mathbb{R}$ is such that

$$
\left\|F^{\prime}\left(x_{0}\right)^{+}\right\|\left\|F^{\prime}(x)-F^{\prime}(y)\right\| \leq g^{\prime}\left(\|y-x\|+\left\|x-x_{0}\right\|\right)-g^{\prime}\left(\left\|x-x_{0}\right\|\right)
$$

for each $x, y \in B\left(x_{0}, R\right)$. Moreover, define iterations $\left\{s_{k}\right\}$ by

$$
s_{0}=0, s_{k+1}=s_{k}-\frac{\varphi_{\eta, \mu}\left(s_{k}\right)}{f_{0}^{\prime}\left(s_{k}\right)} .
$$

This iteration was used by us in [5]. In view of these conditions, we have

$$
f_{0}^{\prime}(t) \leq g^{\prime}(t)
$$

and

$$
f^{\prime}(t) \leq g^{\prime}(t)
$$

for each $t \in\left[0, R^{*}\right)$. Next, the main semi-local convergence result for the Gauss-Newton method is presented. 
Theorem 2.1. Suppose that the $(\mathscr{C})$ conditions hold and $f_{0}^{\prime}(t) \leq f^{\prime}(t)$ for each $t \in\left[0, R^{*}\right]$. Then, the following hold: $\varphi_{\eta, \mu}(t)$ has a smallest zero $r^{*} \in\left(0, R^{*}\right)$, the sequences $\left\{r_{k}\right\}$ and $\left\{x_{k}\right\}$ for solving $\varphi_{\eta, \mu}(t)=0$ and $F(x)=0$, with starting point $t_{0}=0$ and $x_{0}$, respectively given by (1.2) and (2.3) are well defined, $\left\{r_{k}\right\}$ is strictly increasing, remains in $\left[0, r^{*}\right)$, and converges to $r^{*},\left\{x_{k}\right\}$ remains in $B\left(x_{0}, r^{*}\right)$, converges to a point $x^{*} \in B\left(x_{0}, r^{*}\right)$ such that $F^{\prime}\left(x^{*}\right)^{\dagger} F\left(x^{*}\right)=0$. Moreover, the following estimates hold:

$$
\begin{gathered}
\left\|x_{k+1}-x_{k}\right\| \leq r_{k+1}-r_{k} \text { for each } k=0,1,2, \cdots, \\
\left\|x^{*}-x_{k}\right\| \leq r^{*}-r_{k} \text { for each } k=0,1,2, \cdots,
\end{gathered}
$$

and

$$
\left\|x_{k+1}-x_{k}\right\| \leq \frac{r_{k+1}-r_{k}}{\left(r_{k}-r_{k-1}\right)^{2}}\left\|x_{k}-x_{k-1}\right\|^{2} \text { for each } k=0,1,2, \cdots .
$$

Furthermore, if $\mu=0\left(\mu=0\right.$ and $\left.f_{0}^{\prime}\left(r^{*}\right)<0\right)$, the sequence $\left\{r_{k}\right\},\left\{x_{k}\right\}$ converge $Q$-linearly and $R$-linearly $(Q$ - quadratically and $R-$ quadratically) to $r^{*}$ and $x^{*}$, respectively.

Proof. Simply repeat the proof of Theorem 3.9 in [5] (or the proof in [14]) with $f$ replacing $g$. Notice also that the iterates $x_{n}$ remain in $B\left(x_{0}, R_{0}\right)$ which is a more precise location than $B\left(x_{0}, R^{*}\right)$ used in $[5,14]$.

Remark 2.2. (i) As noted in [14] the best choice for $\mu$ is given by $\mu=-\kappa f^{\prime}(\kappa)$.

(ii) If $f(t)=g(t)=f_{0}(t)$ for each $t \in\left[0, R_{0}\right)$ and $R_{0}=R$, then Theorem 2.1 reduces to the corresponding Theorem in [8]. Moreover, if $\left.f_{0}^{\prime}(t) \leq f^{\prime} t\right)=g^{\prime}(t)$ we obtain the results in [5]. If

$$
f_{0}^{\prime}(t) \leq f^{\prime}(t) \leq g^{\prime}(t) \text { for each } t \in\left[0, R^{*}\right)
$$

then the following advantages denoted by $(\mathscr{A})$ are obtained: weaker sufficient convergence criteria, tighter error bounds on the distances $\left\|x_{n}-x^{*}\right\|,\left\|x_{n+1}-x_{n}\right\|$ and an at least as precise information on the location of the solution $x^{*}$. These advantages are obtained using less computational cost, since in practice the computation of function $g$ requires the computation of functions $f_{0}$ and $f$ as special cases. It is also worth noticing that under $\left(c_{1}\right)$ function $f_{0}^{\prime}$ is defined and therefore $R^{*}$ which is at least as small as $R$.

We have that, if function $\bar{\varphi}_{\eta, \mu}$ has a solution $t^{*}$, then, since $\varphi_{\eta, \mu}\left(t^{*}\right) \leq \bar{\varphi}_{\eta, \mu}\left(t^{*}\right)=0$ and $\varphi_{\eta, \mu}(0)=\bar{\varphi}_{\eta, \mu}(0)=\eta>0$, we get that function $\varphi_{\eta, \mu}$ has a solution $r^{*}$ such that

$$
r^{*} \leq t^{*}
$$

but not necessarily vice versa. It also follows from (2.6) that the new information about the location of the solution $x^{*}$ is at least as precise as the one given in [14, 5].

Let us specialize conditions $(\mathscr{C})$ even further in the case when $f_{0}, f$ and $g$ are constant functions $L_{0}, K, L$, respectively. Then, (for $\left.\mu=0\right)$ we have that:

$$
\bar{\varphi}_{\eta, \mu}(t)=\frac{L}{2} t^{2}-t+\eta
$$

and

$$
\varphi_{\eta, \mu}(t)=\frac{K}{2} t^{2}-t+\eta,
$$

respectively. In this case the convergence criteria become, respectively

$$
h=L \eta \leq \frac{1}{2}
$$

and

Notice that

$$
h_{1}=K \eta \leq \frac{1}{2}
$$

$$
h \leq \frac{1}{2} \Longrightarrow h_{1} \leq \frac{1}{2}
$$

but not vice versa unless, $K=$ L. Criterion (2.8) is famous for its simplicity and clarity Kantorovich hypothesis for the semilocal convergence of Newton's method to a solution $x^{*}$ of nonlinear equation $F(x)=0$ [7, 16]. In the case of Wang's conditions [20] we have for $\mu=0$ :

$$
\begin{aligned}
g(t)=\frac{\gamma t^{2}}{1-\gamma t}-t, f(t) & =\frac{\beta t^{2}}{1-\beta t}-t, f_{0}(t)=\frac{\gamma_{0} t^{2}}{1-\gamma_{0} t}-t, \\
\bar{\varphi}_{\eta, \mu}(t) & =\frac{\gamma t^{2}}{1-\gamma t}-t+\eta \\
\varphi_{\eta, \mu}(t) & =\frac{\beta t^{2}}{1-\beta t}-t+\eta
\end{aligned}
$$

with convergence criteria, given respectively by

$$
\begin{gathered}
H=\gamma \eta \leq 3-2 \sqrt{2} \\
H_{1}=\beta \eta \leq 3-2 \sqrt{2} .
\end{gathered}
$$


Then, again we have that

$$
H \leq 3-2 \sqrt{2} \Longrightarrow H_{1} \leq 3-2 \sqrt{2}
$$

but not necessarily vice versa, unless if $\beta=\gamma$.

Concerning the error bounds and the limit of majorizing sequence, suppose that

$$
-\frac{\varphi_{\eta, \mu}(r)}{f_{0}^{\prime}(r)} \leq-\frac{\varphi_{\eta, \mu}(s)}{f_{0}^{\prime}(s)}
$$

for each $r, s \in\left[0, R^{*}\right]$ with $r \leq s$. According to the proof of Theorem 2.1, sequence $\left\{r_{n}\right\}$ is also a majorizing sequence for (1.2). Moreover, a simple induction argument shows that

$$
r_{n} \leq s_{n}, r_{n+1}-r_{n} \leq s_{n+1}-s_{n}
$$

and

$$
r^{*}=\lim _{n \longrightarrow \infty} r_{n} \leq s^{*}
$$

Furthermore, the first two preceding inequalities are strict, for $n \geq 2$ if $f_{0}^{\prime}(t)<f^{\prime}(t)$ for each $t \in\left[0, R^{*}\right]$. Similarly, suppose that

$$
-\frac{\varphi_{\eta, \mu}(s)}{f_{0}^{\prime}(s)} \leq-\frac{\varphi_{\eta, \mu}(t)}{f_{0}^{\prime}(t)}
$$

for each $s, t \in\left[0, R^{*}\right]$ with $s \leq t$. Then, we have that

$$
s_{n} \leq t_{n}, s_{n+1}-s_{n} \leq t_{n+1}-t_{n}
$$

The first two preceding inequalities are also strict for $n \geq 2$, if strict inequality holds in (2.12).

Finally, the rest of the results in $[5,14]$ can be improved along the same lines by also using $K$ instead of $L$. We leave the details to the motivated reader.

\section{Numerical examples}

We present a simple example where we show that Wang's condition (2.11) [20] is violated but our condition (2.12) is satisfied. More examples can be found in [7] where $L_{0} \leq K \leq L$ are satisfied as strict inequalities (therefore the new advantages apply) (or see also [19]).

Example 3.1. Let $\mu=0, p \in(0,1), x_{0}=1, \Omega=B\left(x_{0}, \frac{1}{2-p}\right)$ and define functions on $\Omega$ by

$$
f(x)=\frac{x^{4}}{4}-p x, F(x)=x^{3}-p
$$

Define $\Omega^{*}=B\left(x_{0}, 1-p\right)$. Then, we have

$$
\Omega^{*} \subseteq \Omega, \text { if } p \in[0.381966,1) .
$$

Let $L_{0}=3-p$ and $L=2(2-p)$. Then, Argyros showed in [8] that for each $x, y \in \Omega$

$$
\left|F^{\prime}\left(x_{0}\right)^{-1}\left(F^{\prime}(x)-F^{\prime}\left(x_{0}\right)\right)\right| \leq L_{0}\left|x-x_{0}\right|
$$

and

$$
\left|F^{\prime}\left(x_{0}\right)^{-1}\left(F^{\prime}(x)-F^{\prime}(y)\right)\right| \leq L|x-y|
$$

Consider the conditions

$$
\left\|F^{\prime}\left(x_{0}\right)^{-1} F^{\prime \prime}(x)\right\| \leq \frac{2 \gamma}{\left(1-\gamma\left\|x-x_{0}\right\|\right)^{3}}
$$

for each $x \in \Omega$,

$$
\left\|F^{\prime}\left(x_{0}\right)^{-1}\left(F^{\prime}(x)-F^{\prime}\left(x_{0}\right)\right)\right\| \leq \frac{1}{\left(1-\gamma_{0}\left\|x-x_{0}\right\|\right)^{2}}-1
$$

for each $x \in \Omega$ and

$$
\left\|F^{\prime}\left(x_{0}\right)^{-1} F^{\prime \prime}(x)\right\| \leq \frac{2 \beta}{\left(1-\beta\left\|x-x_{0}\right\|\right)^{3}}
$$

for each $x \in \Omega^{*}$. Notice that functions $\bar{\varphi}_{\eta, 0}, \varphi_{\eta, 0}$ satisfy these conditions, respectively. In view of (3.4) and (3.5), we have $L \leq 2 \gamma$, so we choose $\gamma=2-p$. Then, since $\eta=\frac{1}{3}(1-p)$, condition (2.11) is satisfied, if

$$
0.6255179 \leq p<1 \text {. }
$$

We must have

$$
B\left(x_{0},\left(1-\frac{1}{\sqrt{2}}\right) \frac{1}{\gamma}\right) \subseteq B\left(x_{0}, 1-p\right)
$$


which is true for

$$
0<p \leq 0.7631871
$$

It follows from (3.8) and (3.9) that

$$
0.6255179<p \leq 0.7631871 .
$$

Set $y=\gamma_{0}\left|x-x_{0}\right|$ and $L_{0}=d \gamma_{0}, d>0, \gamma_{0}>0$. Using (3.6) and (3.3), we must have

$$
L_{0}\left|x-x_{0}\right| \leq \frac{1}{\left(1-\gamma_{0}\left|x-x_{0}\right|\right)^{2}}-1
$$

or

$$
d(1-y)^{2} \leq 2-y
$$

or

$$
d y^{2}+(1-2 d) y+d-2 \leq 0
$$

Let e.g. $d=2$, then $\gamma_{0}=\frac{L_{0}}{2}=\frac{3-p}{2}$ and (3.11) becomes $(p-3)(p-1) \leq 3$ or $p(p-4) \leq 0$, which is true. We must show $\left(1-\frac{1}{\sqrt{2}}\right) \frac{1}{\gamma_{0}} \leq 1-p$ or $p^{2}-4 p+1+\sqrt{2} \geq 0$, which is true for

$$
0<p \leq 0.7407199 \text {. }
$$

Notice that $\Omega_{0} \subset \Omega$, since $\left(1-\frac{1}{\sqrt{2}}\right) \frac{1}{\gamma_{0}}<\frac{1}{\gamma}$ or $p \leq 3+\sqrt{2}$, which is true, so

$$
\Omega \cap \Omega_{0}=\Omega_{0} .
$$

Then, for $x \in \Omega_{0}$

$$
\begin{aligned}
\left|F^{\prime}\left(x_{0}\right)^{-1} F^{\prime \prime}(x)\right| & =2|x| \leq 2\left(\left|x-x_{0}\right|+\left|x_{0}\right|\right) \\
& \leq 2\left(\left(1-\frac{1}{\sqrt{2}}\right) \frac{2}{3-p}+1\right)
\end{aligned}
$$

must be smaller than $2 \beta$, so we can choose

$$
\beta=1+\left(1-\frac{1}{\sqrt{2}}\right) \frac{2}{3-p}=1+\frac{2-\sqrt{2}}{3-p} .
$$

Notice that $\beta<\gamma$, if (3.12) holds. We also have that $\gamma_{0}<\beta$, if

$$
\frac{3-p}{2}<1+\frac{2-\sqrt{2}}{3-p}
$$

or if

$$
p^{2}-4 p-1+2 \sqrt{2}<0
$$

or, if

$$
0.5263741<p<1 \text {. }
$$

We also must have

$$
\left(1-\frac{1}{\sqrt{2}}\right) \frac{1}{\beta} \leq 1-p
$$

or

$$
2 p^{2}+(\sqrt{2}-10) p+4+\sqrt{2} \leq 0,
$$

which is true for

$$
p \leq 0.767996
$$

Then, notice that

$$
1-p \leq \frac{1}{\gamma}
$$

if $p^{2}-3 p+1 \leq 0$, which is true for

$$
0.381966 \leq p<1 .
$$

Then, we have that $\alpha_{0} \leq 3-2 \sqrt{2}=q$, if $\left(1+\frac{2-\sqrt{2}}{3-p}\right) \frac{1}{3}(1-p) \leq q$ or if

$$
p^{2}+(\sqrt{2}-6+3 q) p+5-\sqrt{2}-9 q \leq 0
$$

which is true for

$$
0.5857931 \leq p<1
$$


In view of (3.12), (3.14), (3.15) and (3.17) we must have

$$
0.5857931 \leq p \leq 0.7407199 .
$$

Define intervals I and $I_{1}$ by

$$
I=[0.5857931,0.6255179)
$$

and

$$
I_{1}=(0.7407199,0.7631871]
$$

In view of (3.10), (3.19) and (3.20), we see that for $p \in I$ [20] cannot guarantee the convergence of $x_{n}$ to $x^{*}=\sqrt[3]{p}$. However, our Theorem 2.1 guarantees the convergence of $x_{n}$ to $x^{*}$. Notice that, if $p \in I_{1}$, then we can set $\beta=\gamma=\gamma_{0}$.

Next, we compare the error bounds. Choose $p=0.623$. Then, we have the following comparison table, which shows that the new error bounds are more precise than the ones in [20].

\begin{tabular}{|c||c|c|}
\hline \hline$n$ & $r_{n+1}-r_{n}$ & $t_{n+1}-t_{n}$ \\
\hline 1 & 0.1257 & 0.1257 \\
2 & 0.0268 & 0.0333 \\
3 & 0.0013 & 0.0027 \\
4 & $3.3384 \mathrm{e}-06$ & $1.8199 \mathrm{e}-05$ \\
5 & $2.0876 \mathrm{e}-11$ & $8.2197 \mathrm{e}-10$ \\
\hline
\end{tabular}

Table 1: Comparison table.

\section{References}

[1] Argyros, I.: On the semilocal convergence of the Gauss-Newton method. Adv. Nonlinear Var.Inequal. 8(2), 93-99, 2005.

[2] Argyros, I., Hilout, S.: On the local convergence of the Gauss-Newton method. Punjab Univ. J.Math. 41, 23-33, 2009.

[3] Argyros, I., Hilout, S.: On the Gauss-Newton method. J. Appl. Math. Comput. 1-14, 2010.

[4] Argyros, I. K, Hilout, S.: Extending the applicability of the Gauss-Newton method under average Lipschitz-type conditions. Numer. Algorithms 58(1), 23-52, 2011

[5] Argyros, I. K, S. George, Expanding the applicability of the Gauss-Newton method for a certain class of systems of equations, J. Numer.Anal. Approx. Theory, vol., 45(1), 3-13, (2016).

[6] Argyros, I. K., Hilout, S.: Improved local convergence of Newton's method under weak majorant condition, Journal of Computational and Applied Mathematics, 236(7), 1892-1902, 2012.

[7] Argyros, I., Hilout, S.: Numerical methods in nonlinear analysis, World Scientific Publ. Comp. New Jersey, USA, 2013.

[8] Ben-Israel, A., Greville, T.N.E.: Generalized inverses. CMS Books in Mathematics/Ouvrages de Mathematiques de la SMC, 15. Springer-Verlag, New York, second edition, Theory and Applications, 2003.

[9] Catinas, E.: The inexact, inexact perturbed, and quasi-Newton methods are equivalent models, Math. Comput. 74, 249, (2005), $291-301$.

[10] Dedieu, J.P., Kim, M.H.: Newton's method for analytic systems of equations with constant rank derivatives. J. Complexity, $18(1)$ : $187-209,2002$.

[11] Ferreira, O.P., Gonçalves, M.L.N, Oliveira, P.R.:, Local convergence analysis of inexact Gauss-Newton like methods under majorant condition, J. Complexity, 27(1), 111-125, 2011.

[12] Ferreira, O.P., Svaiter, B.F.: Kantorovich's majorants principle for Newton's method. Comput.Optim. Appl. 42(2), 213-229, 2009.

[13] Häussler, W.M.: A Kantorovich-type convergence analysis for the Gauss-Newton-method. Numer. Math. 48(1), 119-125, 1986.

[14] Gonçalves, M.L.N, Oliveira, P.R.:, Convergence of the Gauss- Newton method for a special class of systems of equations under a majorant condition, Optimization, 64, 3(2015), 577-594.

[15] Hu, N., Shen, W. and Li, C.: Kantorovich's type theorems for systems of equations with constant rank derivatives, J. Comput. Appl.Math., 219(1): $110-122,2008$

[16] Kantorovich, L.V., Akilov, G.P., Functional Analysis, Pergamon Press, Oxford, 1982.

[17] Li, C., Hu, N., Wang, J.: Convergence behavior of Gauss-Newton's method and extensions of the Smale point estimate theory. J. Complex. 26(3), $268-295,2010$.

[18] Potra, F.A., Ptak, V.: Nondiscrete induction and iterative processes. Research notes in Mathematics, 103, Pitman(Aóvanced Publishing Program), Boston, MA, 1984

[19] Smale, S., Newton's method estimates from data at one point. The merging of disciplines: new directions in pure, applied, and computational mathematics (Laramie, Wyo., 1985), 185-196, Springer, New York, 1986.

[20] Wang, X.H., Convergence of Newton's method and uniqueness of the solution of equations in Banach spaces, IMA J. Numer. Anal., 20 , 123-134, 2000. 